\title{
Fully Differential Vector-Boson-Fusion Higgs Production at Next-to-Next-to-Leading Order
}

\author{
Matteo Cacciari, ${ }^{1,2,3,4}$ Frédéric A. Dreyer, ${ }^{2,3,4}$ Alexander Karlberg, ${ }^{5}$ Gavin P. Salam, ${ }^{4,}$ and Giulia Zanderighi ${ }^{4,5}$ \\ ${ }^{1}$ Université Paris Diderot, F-75013 Paris, France \\ ${ }^{2}$ Sorbonne Universités, UPMC Université Paris 06, UMR 7589, LPTHE, F-75005 Paris, France \\ ${ }^{3}$ CNRS, UMR 7589, LPTHE, F-75005 Paris, France \\ ${ }^{4}$ CERN, PH-TH, CH-1211 Geneva 23, Switzerland \\ ${ }^{5}$ Rudolf Peierls Centre for Theoretical Physics, 1 Keble Road, University of Oxford, Oxford OX1 3NP, United Kingdom
}

(Received 16 June 2015; published 21 August 2015)

\begin{abstract}
We calculate the fully differential next-to-next-to-leading-order (NNLO) corrections to vector-boson fusion (VBF) Higgs boson production at proton colliders, in the limit in which there is no cross talk between the hadronic systems associated with the two protons. We achieve this using a new "projection-to-Born" method that combines an inclusive NNLO calculation in the structure-function approach and a suitably factorized next-to-leading-order VBF Higgs plus three-jet calculation, using appropriate Higgs plus twoparton counterevents. An earlier calculation of the fully inclusive cross section had found small NNLO corrections, at the $1 \%$ level. In contrast, the cross section after typical experimental VBF cuts receives NNLO contributions of about (5-6)\%, while differential distributions show corrections of up to (10-12)\% for some standard observables. The corrections are often outside the next-to-leading-order scale-uncertainty band.
\end{abstract}

DOI: 10.1103/PhysRevLett.115.082002

PACS numbers: $12.38 . \mathrm{Bx}, 14.80 . \mathrm{Bn}$

Following the discovery in 2012 of the Higgs boson $[1,2]$, one of the main tasks for particle physics today is the accurate determination of its properties and couplings. For the coming decade at least, these studies will take place at CERN's Large Hadron Collider (LHC).

The most relevant production channels for the Higgs boson at the LHC are gluon fusion $(g g H)$, vector-boson fusion $(\mathrm{VBFH})$, production in association with a vector boson $(\mathrm{VH})$, and with a top-quark pair $(t t H)$ [3]. VBFH is special for a number of reasons $[4,5]$ : it has the largest cross section of the processes that involves tree-level production of the Higgs boson (and is second largest among all processes); it has a distinctive signature of two forward jets, which makes it possible to tag the events and so identify Higgs decays that normally have large backgrounds, e.g., $H \rightarrow \tau^{+} \tau^{-}$; the Higgs transverse momentum is nonzero even at lowest order, which facilitates searches for invisible decay modes [6,7]; and it also brings particular sensitivity to the charge-parity properties of the Higgs boson, and nonstandard Higgs interactions, through the angular correlations of the forward jets [8].

Given the key role of VBF Higgs-boson production at the LHC, it is of paramount importance to have a precise prediction for its production. The total cross section was calculated to next-to-next-to-leading-order (NNLO) in Refs. $[9,10]$ using the structure-function approach [11],

Published by the American Physical Society under the terms of the Creative Commons Attribution 3.0 License. Further distribution of this work must maintain attribution to the author(s) and the published article's title, journal citation, and DOI. showing small corrections relative to the next-to-leadingorder (NLO) and tiny renormalization and factorization scale uncertainties, well below $1 \%$. However, experimental measurements are necessarily restricted to a subset of phase space. In particular, because of their use of transversemomentum cuts on the forward tagging jets, one might imagine that there are important NNLO corrections, associated with those jet cuts, that would not be seen in a fully inclusive calculation. Currently, the fully differential VBFH cross section is known only to NLO [12]. It appears to have small scale uncertainties.

One can think of the VBFH process, represented at the Born level in Fig. 1(a), as involving two deeply inelastic scatterings (DIS), one for each of the incoming protons. Each DIS process produces a vector boson, $W^{ \pm}$or $Z$, and the fusion of the vector bosons produces a Higgs boson. The structure-function approach [11] used for the NNLO total cross section $[9,10]$ assumes that the upper and lower hadronic sectors factorize from each other, i.e., that there is no cross talk between them. Factorization is believed to be accurate to better than $1 \%$ in the experimentally relevant kinematic region [10,13-15]. (The factorization of the two sectors is exact if one imagines two copies of QCD, $\mathrm{QCD}_{1}$ and $\mathrm{QCD}_{2}$, respectively, for the upper and lower sectors, where each of the two QCD copies interacts with the electroweak sector, but not with the other QCD copy. This observation could be exploited, for example, in automated calculations and for determining corrections beyond the factorized approximation.)

The reason that the structure-function approach does not provide a fully differential cross section is related to the fact that the DIS coefficient functions used in the calculation 
(a)

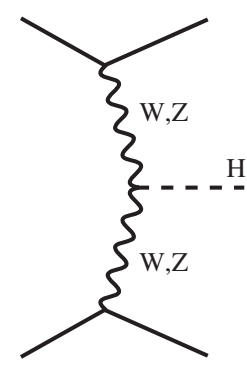

(b)

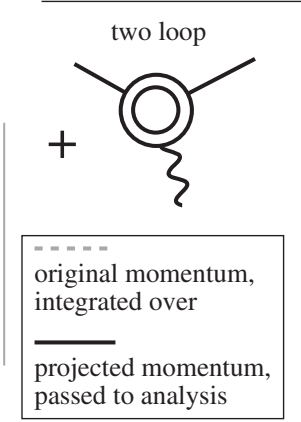

(c)

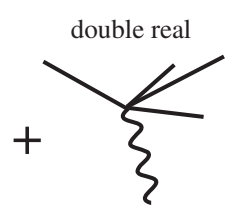

one-loop single real
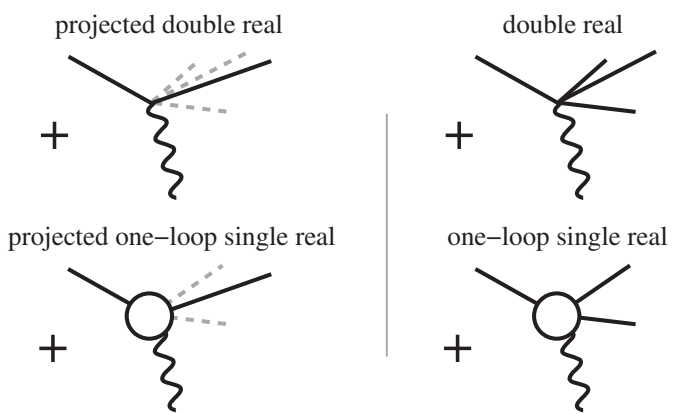

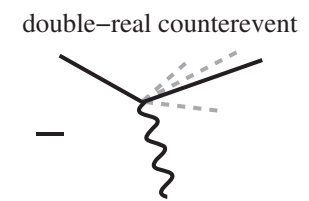

one-loop single-real counterevent

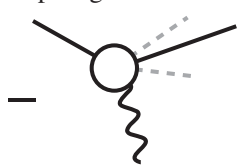

FIG. 1. (a) Illustration of the Born VBFH process. (b) NNLO corrections to the upper sector of the VBF process, from the "inclusive" part of our calculation. (c) Corresponding "exclusive" part. The double-real and one-loop single-real counterevents in the exclusive part cancel the projected double-real and one-loop single-real contributions in the inclusive part. In the "projected" and "counterevent" contributions, the dashed lines corresponds to the full set of parton momenta that are integrated over (for the structure functions, this integral is implicit in the derivation of the coefficient functions), while the solid lines correspond to the partons that are left over after projection to Born-like kinematics and then passed to the analysis. The projection does not change the direction of initial partons and so the corresponding incoming dashed lines are implicit.

implicitly integrate over hadronic final states. In this Letter, we introduce a new "projection-to-Born" approach to eliminate this limitation, and thus provide the first fully differential NNLO calculation of VBF Higgs production in the factorized approximation.

Let us start by recalling that the cross section in the structure-function approach is expressed [9-11] as a sum of terms involving products of structure functions, e.g., $F_{2}\left(x_{1}, Q_{1}^{2}\right) F_{2}\left(x_{2}, Q_{2}^{2}\right)$, where $Q_{i}^{2}=-q_{i}^{2}>0$ is given in terms of the four-momentum $q_{i}$ of the (outgoing) exchanged vector boson $i$. The $x_{i}$ values are fixed by the relation $x_{i}=-Q_{i}^{2} /\left(2 P_{i} \cdot q_{i}\right)$, where $P_{i}$ is the momentum of proton $i$. To obtain the total cross section, one integrates over all $q_{1}, q_{2}$ that can lead to the production of a Higgs boson. If the underlying upper (lower) scattering is Bornlike, quark $\rightarrow$ quark $+V$, then it is straightforward to show that knowledge of the vector-boson momentum $q_{1}\left(q_{2}\right)$ uniquely determines the momenta of both the incoming and outgoing (on-shell) quarks,

$$
p_{\text {in }, i}=x_{i} P_{i}, \quad p_{\text {out }, i}=x_{i} P_{i}-q_{i} .
$$

We exploit this feature in order to assemble a full calculation from two separate ingredients. For the first one, the "inclusive" ingredient, we remain within the structure-function approach, and for each set of $q_{1}$ and $q_{2}$ use Eq. (1) to assign VBF Born-like kinematics to the upper and lower sectors. This is represented in Fig. 1(b) (showing, for brevity, just the upper sector): for the two-loop contribution, the Born kinematics that we assign corresponds to that of the actual diagrams; for the tree-level double-real and one-loop single-real diagrams, it corresponds to a projection from the true kinematics ( $2 \rightarrow H+n$ for $n=3$, 4) down to the Born kinematics $(2 \rightarrow H+2)$. The projected momenta are used to obtain the inclusive contribution to differential cross sections. Note that the Higgs momentum is unaffected by the projection.
Our second, "exclusive," ingredient starts from the NLO fully differential calculation of vector-boson fusion Higgs production with three jets [16,17], as obtained in a factorized approximation, i.e., where there is no cross talk between upper and lower sectors. (The NLO calculation without this approximation is given in Ref. [18].) Thus each parton can be uniquely assigned to one of the upper or lower sectors and the two vector-boson momenta can be unambiguously determined. For each event in a Monte Carlo integration over phase space, with weight $w$, we add a counterevent, with weight $-w$, to which we assign projected Born VBF kinematics based on the vectorboson momenta and Eq. (1). This is illustrated in Fig. 1(c). From the original events, we thus obtain the full momentum structure for tree-level double-real and one-loop single-real contributions. Meanwhile, after integration over phase space, the counterevents exactly cancel the projected tree-level double-real and one-loop single-real contributions from the inclusive part of the calculation. Thus, the sum of the inclusive and exclusive parts gives the complete differential NNLO VBFH result. (Our approach can be contrasted with the differential NNLO structure-function type calculation for single-top production [19] in that we do not need any fully differential ingredients at NNLO.)

For the implementation of the inclusive part of the calculation, we have taken the phase space from POWHEG's Higgs plus two-jet VBF calculation [20], while the matrix element has been coded with structure functions evaluated using parametrized versions [21,22] of the NNLO DIS coefficient functions [23-25] integrated with version 1.1.5 of HOPPET [26]. We have tested our implementation against the results of one of the codes used in Refs. $[9,10]$ and found agreement, both for the structure functions and the final cross sections. We have also checked that switching to the exact DIS coefficient functions has a negligible impact. A further successful comparison of the evaluation of structure functions was made against version 2.4.1 of APFEL [27]. 
For the exclusive part of the calculation, as a starting point we took the NLO (i.e., fixed-order, but not parton-shower) part of the POWHEG $H+$ three-jet VBF code [17], itself based on the calculation of Ref. [16], with tree-level matrix elements from MadGraph 4 [28]. This code already uses a factorized approximation for the matrix element, however, for a given phase-space point it sums over matrix-element weights for the assignments of partons to upper and lower sectors. We therefore reengineered the code so that for each set of four momenta, weights are decomposed into the contributions for each of the different possible sets of assignments of partons to the two sectors. For every element of this decomposition it is then possible to unambiguously obtain the vector-boson momenta and so correctly generate a counterevent. The POWHEG-BOX's [29,30] "tagging" facility was particularly useful in this respect, notably for the NLO subtraction terms. To check the correctness of the assignment to sectors, we verified that as the rapidity separation between the two leading jets increases, there was a decreasing relative fraction of the cross section for which partons assigned to the upper (lower) sector were found in the rapidity region associated with the lower (upper) leading jet. We also tested that the sum of inclusive and exclusive contributions at NLO agrees with the POWHEG NLO implementation of the VBF $H+$ two-jet process.

To investigate the phenomenological consequences of the NNLO corrections, we study $13 \mathrm{TeV}$ proton-proton collisions. We use a diagonal Cabibbo-Kobayashi-Maskawa matrix, full Breit-Wigner distributions for the $W, Z$, and the narrow-width approximation for the Higgs boson. We take NNPDF 3.0 parton distribution functions at NNLO with $\alpha_{s}\left(M_{Z}\right)=0.118$ (NNPDF30_nnlo_as_0118) [31], also for our leading-order (LO) and NLO results. We have five light flavors and ignore contributions with top quarks in the final state or internal lines. We set the Higgs mass to $M_{H}=125 \mathrm{GeV}$, compatible with the experimentally measured value [32]. Electroweak parameters are set according to known experimental values and tree-level electroweak relations. As inputs we use $M_{W}=80.398 \mathrm{GeV}, M_{Z}=$ $91.1876 \mathrm{GeV}$ and $G_{F}=1.16637 \times 10^{-5} \mathrm{GeV}^{-1}$. For the widths of the vector bosons we use $\Gamma_{W}=2.141 \mathrm{GeV}$ and $\Gamma_{Z}=2.4952 \mathrm{GeV}$.

Some care is needed with the renormalization and factorization scale choice. A natural option would be to use $Q_{1}$ and $Q_{2}$ as our central values for the upper and lower sectors, respectively. While this is straightforward in the inclusive code, in the exclusive code we had the limitation that the underlying POWHEG-BOX code can presently only easily assign a single scale (or set of scales) to a given event. However, for each POWHEG phase-space point, we have multiple assignments of partons to the upper and lower sectors, leading to several $\left\{Q_{1}, Q_{2}\right\}$ pairs for each event. Thus the use of $Q_{1}$ and $Q_{2}$ would require some further degree of modification of the POWHEG-BOX, which we leave to future work. We instead choose a central scale that depends on the Higgs transverse momentum $p_{t, H}$ :

$$
\mu_{0}^{2}\left(p_{t, H}\right)=\frac{M_{H}}{2} \sqrt{\left(\frac{M_{H}}{2}\right)^{2}+p_{t, H}^{2}} .
$$

This choice of $\mu_{0}$ is usually close to $\sqrt{Q_{1} Q_{2}}$. It represents a good compromise between satisfying the requirement of a single scale for each event, while dynamically adapting to the structure of the event. In order to estimate missing higher-order uncertainties, we vary the renormalization and factorization scales symmetrically (i.e., keeping $\mu_{R}=\mu_{F}$ ) by a factor of 2 up and down around $\mu_{0}$. (We verified that an expanded scale variation, allowing $\mu_{R} \neq \mu_{F}$ with $\frac{1}{2}<\mu_{R} / \mu_{F}<2$, led only to very small changes in the NNLO scale uncertainties for the VBF-cut cross section and the $p_{t, H}$ distribution.)

To pass our VBF selection cuts, events should have at least two jets with transverse momentum $p_{t}>25 \mathrm{GeV}$; the two hardest (i.e., highest $p_{t}$ ) jets should have absolute rapidity $|y|<4.5$, be separated by a rapidity $\Delta y_{j_{1}, j_{2}}>4.5$, have a dijet invariant mass $m_{j_{1}, j_{2}}>600 \mathrm{GeV}$, and be in opposite hemispheres $\left(y_{j_{1}} y_{j_{2}}<0\right)$. Jets are defined using the anti- $k_{t}$ algorithm [33], as implemented in version 3.1.2 of FastJet [34], with radius parameter $R=0.4$.

Results are shown in Table I for the fully inclusive cross section and with our VBF cuts. One sees that the NNLO corrections modify the fully inclusive cross section only at the percent level, which is compatible with the findings of Ref. [9]. However, after VBF cuts, the NNLO corrections are about 5 times larger, reducing the cross section by (5-6)\% relative to NLO. The magnitude of the NNLO effects after cuts implies that it will be essential to take them into account for future precision studies. Note that in both the inclusive and VBF-cut cases, the NNLO contributions are larger than would be expected from NLO scale variation.

Differential cross sections are shown in Fig. 2, for events that pass the VBF cuts. From left to right, the plot shows the transverse momentum distributions for the two leading jets $p_{t, j_{1}}$ and $p_{t, j_{2}}$, for the Higgs boson $p_{t, H}$, and the distribution for the rapidity separation between the two leading jets $\Delta y_{j_{1}, j_{2}}$. The bands and the patterned boxes denote the scale uncertainties, while the vertical error bars denote the statistical uncertainty. The effect of the NNLO corrections on the jets appears to be to reduce their transverse momentum, leading to negative (positive) corrections in

TABLE I. Cross sections at LO, NLO, and NNLO for VBF Higgs production, fully inclusively and with VBF cuts. The quoted uncertainties correspond to scale dependence, while statistical errors at NNLO are about $0.1 \%$ with VBF cuts and much smaller without.

\begin{tabular}{lcc}
\hline \hline & $\sigma^{\text {(no cuts })}(\mathrm{pb})$ & $\sigma^{\text {(VBF cuts })}(\mathrm{pb})$ \\
\hline LO & $4.032_{-0.069}^{+0.057}$ & $0.957_{-0.059}^{+0.066}$ \\
NLO & $3.929_{-0.023}^{+0.024}$ & $0.876_{-0.018}^{+0.008}$ \\
NNLO & $3.888_{-0.012}^{+0.016}$ & $0.826_{-0.014}^{+0.013}$ \\
\hline \hline
\end{tabular}



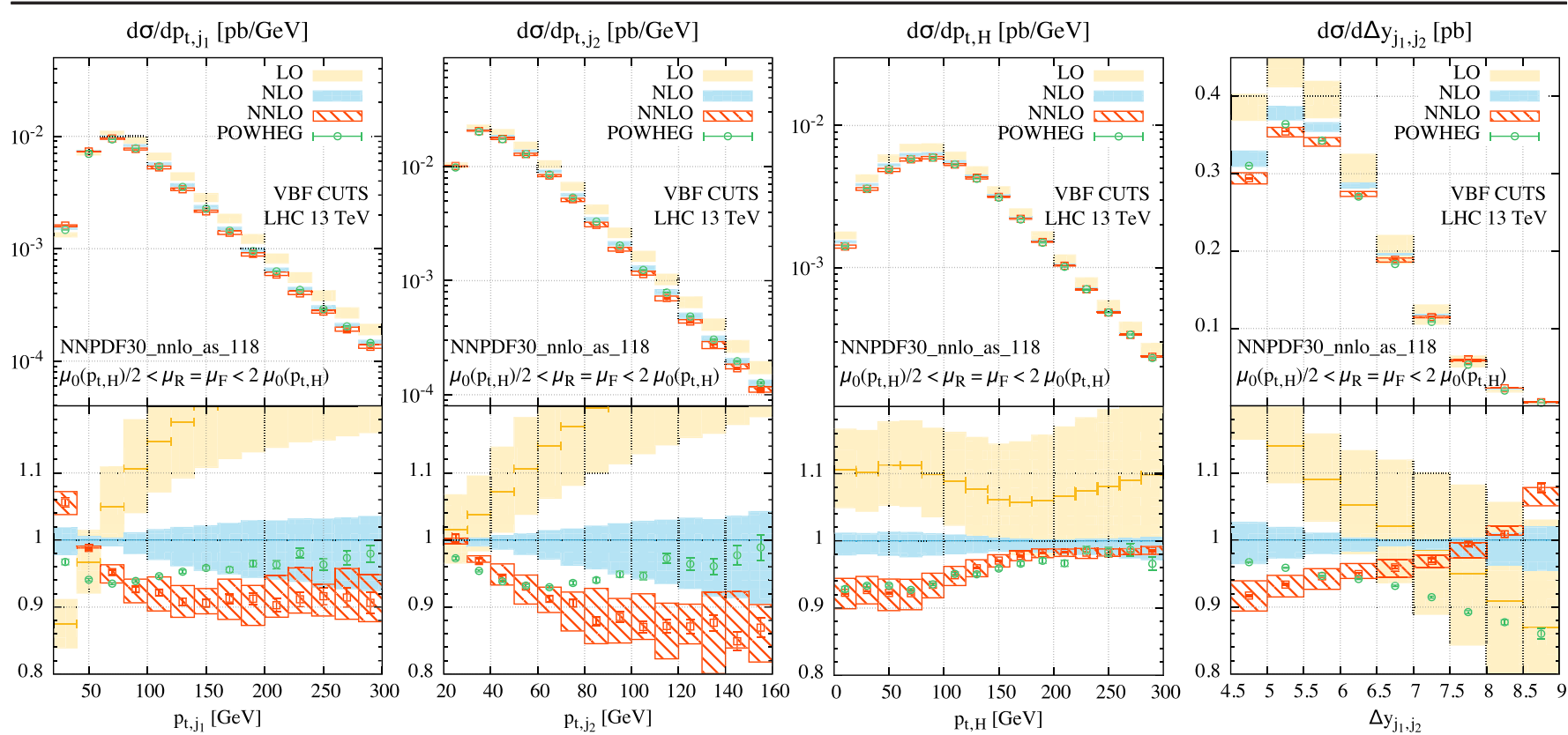

FIG. 2 (color online). From left to right, differential cross sections for the transverse momentum distributions for the two leading jets $p_{t, j_{1}}$ and $p_{t, j_{2}}$, for the Higgs boson $p_{t, H}$, and the distribution for the rapidity separation between the two leading jets $\Delta y_{j_{1}, j_{2}}$.

regions of falling (rising) jet spectra. One can see effects of up to $(10-12) \%$. Turning to $p_{t, H}$, one might initially be surprised that such an inclusive observable should also have substantial NNLO corrections, of about $8 \%$ for low and moderate $p_{t, H}$. Our interpretation is that since NNLO effects redistribute jets from higher to lower $p_{t}$ 's (cf. the plots for $p_{t, j_{1}}$ and $p_{t, j_{2}}$ ), they reduce the cross section for any observable defined with VBF cuts. As $p_{t, H}$ grows larger, the forward jets tend naturally to get harder and so automatically pass the $p_{t}$ thresholds, reducing the impact of NNLO terms.

As observed above for the total cross section with VBF cuts, the NNLO differential corrections are sizable and often outside the uncertainty band suggested by NLO scale variation. One reason for this might be that NLO is the first order where the noninclusiveness of the jet definition matters, e.g., radiation outside the cone modifies the cross section. Thus NLO is, in effect, a leading-order calculation for the exclusive corrections, with all associated limitations.

To further understand the size of the NNLO corrections, it is instructive to examine a NLO plus parton shower (NLOPS) calculation, since the parton shower will include some approximation of the NNLO corrections. For this purpose, we have used the POWHEG VBF $H+$ two-jet calculation [20], showered with PYTHIA version 6.428 with the Perugia 2012 tune [35]. The POWHEG part of this NLOPS calculation uses the same PDF, scale choices, and electroweak parameters as our full NNLO calculation. The NLOPS results are included in Fig. 2, at parton level, with multiparton interactions (MPI) switched off. They differ from the NLO by an amount that is of a similar order of magnitude to the NNLO effects. This lends support to our interpretation that final- (and initial-)state radiation from the hard partons is responsible for a substantial part of the NNLO corrections. However, while the NLOPS calculation reproduces the shape of the NNLO corrections for some observables (especially $p_{t, H}$ ), there are others for which this is not the case, the most striking being perhaps $\Delta y_{j_{1}, j_{2}}$. Parton shower effects were also studied in Ref. [36], using the MC@NLO approach [37]. Various parton showers differed there by up to about $10 \%$.

In addition to the NNLO contributions, precise phenomenological studies require the inclusion of electroweak contributions and nonperturbative hadronization and MPI corrections. The former are of the same order of magnitude as our NNLO corrections [13]. Using PYTHIA version 6.428 and PYTHIA version 8.185 we find that hadronization corrections are between $-2 \%$ and $0 \%$, while MPI brings up to $+5 \%$ at low $p_{t}$ 's. The small hadronization corrections appear to be due to a partial cancellation between shifts in $p_{t}$ and rapidity. We leave a combined study of all effects to future work. The code for our calculation will also be made public.

With the calculation presented in this Letter, differential VBF Higgs production has been brought to the same NNLO level of accuracy that has been available for some time now for the $g g H[38,39]$ and $\mathrm{VH}[40]$ production channels. This constitutes the first fully differential NNLO $2 \rightarrow 3$ hadroncollider calculation, an advance made possible thanks to the factorizable nature of the process. The NNLO corrections are non-negligible, $(5-10) \%$, i.e., an order of magnitude larger than the corrections to the inclusive cross section. Their size might even motivate a calculation one order higher, to NNNLO, to match the precision achieved recently for the $g g H$ total cross section [41]. With the new projectionto-Born approach introduced here, we believe that this is within reach. It would also be of interest to obtain NNLO 
plus parton shower predictions, again matching the accuracy achieved recently in $g g H[42,43]$.

We wish to thank Marco Zaro for sharing his private code for the inclusive NNLO calculation, which enabled valuable cross checks, and for numerous useful discussions as well as comments on the manuscript. We also thank Fabrizio Caola for his careful reading of the manuscript and valuable comments. We are grateful to Paolo Nason for his suggestions concerning the adaptation of the POWHEG code, to Barbara Jäger for helpful exchanges about the VBF Higgs plus three-jet process, to Andreas Vogt for supplying us with the exact coefficient DIS coefficient functions, and to Valerio Bertone and Juan Rojo for discussions about APFEL. We also benefited from discussions with John Ellis, Zoltan Kunszt, and Fabio Maltoni. F. D. is supported by the ILP LABEX (ANR-10-LABX-63) financed by French state funds managed by the ANR within the Investissements d'Avenir programme under reference ANR-11-IDEX0004-02. A. K. is supported by the British Science and Technology Facilities Council and by the Buckee Scholarship at Merton College. This work was supported also by ERC Consolidator Grant HICCUP and by ERC Advanced Grant Higgs@LHC. A. K. thanks CERN for hospitality while part of this work was performed.

* On leave from CNRS, UMR 7589, LPTHE, F-75005 Paris, France.

[1] G. Aad et al. (ATLAS Collaboration), Phys. Lett. B 716, 1 (2012).

[2] S. Chatrchyan et al. (CMS Collaboration), Phys. Lett. B 716, 30 (2012).

[3] S. Dittmaier et al. (LHC Higgs Cross Section Working Group Collaboration), Handbook of LHC Higgs Cross Sections: 1. Inclusive Observables (CERN, Geneva, 2011), p. 153.

[4] S. Dittmaier, S. Dittmaier, C. Mariotti, G. Passarino, R. Tanaka, S. Alekhin, J. Alwall, and E. A. Bagnaschi et al., Handbook of LHC Higgs Cross Sections: 2. Differential Distributions (CERN, Geneva, 2012), p. 275.

[5] S. Heinemeyer et al. (LHC Higgs Cross Section Working Group Collaboration), Handbook of LHC Higgs Cross Sections: 3. Higgs Properties : Report of the LHC Higgs Cross Section Working Group (CERN, Geneva, 2013), p. 404.

[6] (ATLAS Collaboration), Search for an Invisibly Decaying Higgs Boson Produced via Vector Boson Fusion in $p p$ Collisions at $\sqrt{S}=8 \mathrm{TeV}$ Using the ATLAS Detector at the LHC, Report No. ATLAS-CONF-2015-004.

[7] (CMS Collaboration), Search for Invisible Decays of Higgs Bosons in the Vector Boson Fusion Production Mode, Report No. CMS-PAS-HIG-14-038.

[8] T. Plehn, D. L. Rainwater, and D. Zeppenfeld, Phys. Rev. Lett. 88, 051801 (2002).

[9] P. Bolzoni, F. Maltoni, S. O. Moch, and M. Zaro, Phys. Rev. Lett. 105, 011801 (2010).

[10] P. Bolzoni, F. Maltoni, S. O. Moch, and M. Zaro, Phys. Rev. D 85, 035002 (2012).

[11] T. Han, G. Valencia, and S. Willenbrock, Phys. Rev. Lett. 69, 3274 (1992).
[12] T. Figy, D. Zeppenfeld, and C. Oleari, Phys. Rev. D 68, 073005 (2003).

[13] M. Ciccolini, A. Denner, and S. Dittmaier, Phys. Rev. D 77, 013002 (2008).

[14] J. R. Andersen, T. Binoth, G. Heinrich, and J. M. Smillie, J. High Energy Phys. 02 (2008) 057.

[15] See also the summary in M. Zaro, Ph.D. thesis, Université catholique de Louvain, 2013.

[16] T. Figy, V. Hankele, and D. Zeppenfeld, J. High Energy Phys. 02 (2008) 076.

[17] B. Jäger, F. Schissler, and D. Zeppenfeld, J. High Energy Phys. 07 (2014) 125.

[18] F. Campanario, T. M. Figy, S. Platzer, and M. Sjodahl, Phys. Rev. Lett. 111, 211802 (2013).

[19] M. Brucherseifer, F. Caola, and K. Melnikov, Phys. Lett. B 736, 58 (2014).

[20] P. Nason and C. Oleari, J. High Energy Phys. 02 (2010) 037.

[21] W. L. van Neerven and A. Vogt, Nucl. Phys. B568, 263 (2000).

[22] W. L. van Neerven and A. Vogt, Nucl. Phys. B588, 345 (2000).

[23] W. L. van Neerven and E. B. Zijlstra, Phys. Lett. B 272, 127 (1991).

[24] E. B. Zijlstra and W. L. van Neerven, Nucl. Phys. B383, 525 (1992).

[25] E. B. Zijlstra and W. L. van Neerven, Phys. Lett. B 297, 377 (1992).

[26] G. P. Salam and J. Rojo, Comput. Phys. Commun. 180, 120 (2009).

[27] V. Bertone, S. Carrazza, and J. Rojo, Comput. Phys. Commun. 185, 1647 (2014).

[28] J. Alwall, P. Demin, S. de Visscher, R. Frederix, M. Herquet, F. Maltoni, T. Plehn, and D. L. Rainwater, and T. Stelzer, J. High Energy Phys. 09 (2007) 028.

[29] P. Nason, J. High Energy Phys. 11 (2004) 040.

[30] S. Alioli, P. Nason, C. Oleari, and E. Re, J. High Energy Phys. 06 (2010) 043.

[31] R. D. Ball et al. (NNPDF Collaboration), J. High Energy Phys. 04 (2015) 040.

[32] G. Aad et al. (ATLAS and CMS Collaborations), Phys. Rev. Lett. 114, 191803 (2015).

[33] M. Cacciari, G. P. Salam, and G. Soyez, J. High Energy Phys. 04 (2008) 063.

[34] M. Cacciari, G. P. Salam, and G. Soyez, Eur. Phys. J. C 72, 1896 (2012).

[35] P. Z. Skands, Phys. Rev. D 82, 074018 (2010).

[36] S. Frixione, P. Torrielli, and M. Zaro, Phys. Lett. B 726, 273 (2013).

[37] S. Frixione and B. R. Webber, J. High Energy Phys. 06 (2002) 029.

[38] S. Catani and M. Grazzini, Phys. Rev. Lett. 98, 222002 (2007).

[39] C. Anastasiou, K. Melnikov, and F. Petriello, Phys. Rev. Lett. 93, 262002 (2004).

[40] G. Ferrera, M. Grazzini, and F. Tramontano, Phys. Rev. Lett. 107, 152003 (2011).

[41] C. Anastasiou, C. Duhr, F. Dulat, F. Herzog, and B. Mistlberger, Phys. Rev. Lett. 114, 212001 (2015).

[42] K. Hamilton, P. Nason, E. Re, and G. Zanderighi, J. High Energy Phys. 10 (2013) 222.

[43] S. Höche, Y. Li, and S. Prestel, Phys. Rev. D 90, 054011 (2014). 